\title{
New objects with the $B[e]$ phenomenon in the Large Magellanic Cloud ${ }^{\star}$
}

\author{
H. Levato ${ }^{1}$, A. S. Miroshnichenko ${ }^{2}$, and C. Saffe ${ }^{1}$ \\ ${ }^{1}$ Instituto de Ciencias Astronómicas, de la Tierra y del Espacio (ICATE), CONICET, Casilla de Correo 49, CP 5400 San Juan, \\ Argentina \\ e-mail: hlevato@icate-conicet.gob.ar \\ 2 Department of Physics and Astronomy, University of North Carolina at Greensboro, Greensboro NC 27402-6170, USA \\ e-mail: a_mirosh@uncg.edu
}

Received 19 March 2014 / Accepted 3 June 2014

\section{ABSTRACT}

\begin{abstract}
Aims. The study is aimed at discovering new objects with the B[e] phenomenon in the Large Magellanic Cloud. Methods. We report medium-resolution optical spectroscopic observations of two newly found (ARDB54 and NOMAD 0181-0125572) and two previously known (Hen S-59 and Hen S-137) supergiants with the B[e] phenomenon in the Large Magellanic Cloud. The observations were obtained with the GMOS spectrograph at the southern Gemini telescope.

Results. The optical spectra and the fundamental parameters of ARDB 54 and NOMAD 0181-0125572 are presented for the first time. We found that the Balmer line profiles of Hen S-59 and Hen S-137 were different from those observed in their spectra nearly 20 years ago. We suggest a higher effective temperature and luminosity for both objects. With the new fundamental parameters, the lowest luminosity for known supergiants with the $\mathrm{B}[\mathrm{e}]$ phenomenon in the Magellanic Clouds is higher that previously thought $\left(\log L / L_{\odot} \sim 4.5\right.$ instead of 4.0). The object Hen S-59 may be a binary system based on its UV excess, variable $B-V$ color-index and radial velocity of emission lines, and periodically variable $I$-band brightness.
\end{abstract}

Key words. stars: early-type - stars: emission-line, Be - supergiants - techniques: spectroscopic - Magellanic Clouds circumstellar matter

\section{Introduction}

Early-type stars are frequently surrounded by circumstellar (CS) matter, which causes the presence of line emission and IR-excesses in their spectra. In addition to permitted lines that mostly form not far from the stellar surface (e.g., H I), forbidden lines (e.g., [O I] and [Fe II]) may form in the outer parts of extended CS envelopes/disks. The simultaneous presence of both types of lines was discovered in the spectra of 65 Galactic B-type stars nearly 40 years ago (Allen \& Swings 1976) and has been known as the B[e] phenomenon (Conti 1976).

After two decades of studies, it was recognized that this phenomenon can be observed in objects at various evolutionary stages (pre-main-sequence Herbig Ae/Be stars, symbiotic binaries, compact planetary nebulae, and supergiants, Lamers et al. 1998) but with similar conditions in the CS environments. It was also found that nearly half of the originally identified objects with the $\mathrm{B}[\mathrm{e}]$ phenomenon were unclassified. Recently, Miroshnichenko (2007) and Miroshnichenko et al. (2007) suggested that the latter objects are most likely binary systems with B-type to early A-type primaries in a luminosity range of

\footnotetext{
* Based on observations obtained at the Gemini Observatory, which is operated by the Association of Universities for Research in Astronomy, Inc., under a cooperative agreement with the NSF on behalf of the Gemini partnership: the National Science Foundation (United States), the National Research Council (Canada), CONICYT (Chile), the Australian Research Council (Australia), Ministério da Ciência, Tecnologia e Inovacão (Brazil) and Ministerio de Ciencia, Tecnología e Innovación Productiva (Argentina).
}

$\left(\log L / L_{\odot} \sim 2.5-4.5\right)$ near the end of evolution on the mainsequence. These authors did not exclude a more evolved nature of the unclassified group, which they called FS CMa type objects but pointed out to their observational differences with proto-planetary and young planetary nebulae.

Since some objects with the $\mathrm{B}[\mathrm{e}]$ phenomenon are quite luminous, studies of emission-line stars in the Magellanic Clouds led to their discoveries already a decade after the introduction of the phenomenon. The first object (R 126) with the $\mathrm{B}[\mathrm{e}]$ phenomenon in the Magellanic Clouds was reported by Zickgraf et al. (1985). Seven more objects were added by Zickgraf et al. (1986), another three by Stahl et al. (1989) and Zickgraf et al. (1989, 1992), and four more by Gummersbach et al. (1995). Eleven objects reported in these studies were found in the Large Magellanic Cloud (LMC) and four in the Small Magellanic Cloud (SMC). All of them are located beyond the main-sequence and have strong IR excesses due to radiation of CS dust. An overview of the group is given in Zickgraf (2006). Additionally, Graus et al. (2012) found three dust-poor supergiants with the $\mathrm{B}[\mathrm{e}]$ phenomenon in the SMC. No FS CMa type objects have been found in the Clouds yet.

The origin of $\mathrm{B}[\mathrm{e}]$ supergiants is still unclear. Most Galactic objects of this kind were found to be binaries (see discussion in Wang et al. 2012), suggesting that interaction between the components may lead to formation of CS disks. Only one B[e] supergiant binary, LHA 115-S 6 (R 4), was reported in the SMC by Zickgraf (2006). Another one, Hen S-18, in the same galaxy was recently found through analysis of long-term brightness variations, although no orbit has been determined (Clark et al. 2013). 
Table 1. Positions, photometry, and derived parameters of the observed objects.

\begin{tabular}{lcccccccccccc}
\hline \hline Name & RA & Dec & $V$ & $U-B$ & $B-V$ & $K$ & $J-H$ & $H-K$ & $E(B-V)$ & $T_{\text {eff }}$ & $M_{\mathrm{V}}$ & $\log L$ \\
\hline ARDB 54 & $4: 54: 43.5$ & $-70: 21: 27.8$ & 12.77 & -0.21 & 0.25 & 11.55 & 0.34 & 0.81 & 0.15 & 10000 & -6.2 & 4.57 \\
ARDB 54 $^{b}$ & & & 12.71 & -0.14 & 0.22 & & & & & & & \\
ARDB 54 & & & 12.71 & -0.16 & 0.25 & & & & & & \\
N181 & $5: 27: 47.6$ & $-71: 48: 52.6$ & 14.24 & -0.71 & 0.10 & 11.77 & 0.74 & 0.98 & 0.15 & 17000 & -4.7 & 4.45 \\
Hen S-59 $^{a}$ & $5: 45: 29.5$ & $-68: 11: 45.7$ & 14.02 & -1.14 & 0.49 & 11.48 & 0.66 & 0.96 & 0.15 & 19000 & -4.9 & 4.64 \\
Hen S-59 $^{d}$ & & & 14.41 & -1.00 & 0.21 & 11.39 & 0.71 & 1.09 & 0.05 & 14000 & -4.2 & 4.00 \\
Hen S-137 $^{a}$ & $5: 41: 43.7$ & $-69: 37: 38.3$ & 14.11 & -0.69 & 0.09 & 11.14 & 0.90 & 1.10 & 0.15 & 17000 & -4.8 & 4.56 \\
Hen S-137 & & & 14.04 & -0.56 & 0.16 & 11.11 & 0.82 & 1.16 & 0.17 & 13000 & -5.0 & 4.20 \\
\hline
\end{tabular}

Notes. Column information: 1 - object ID; 2,3 - coordinates for the epoch 2000.0; 4-9 - optical and near-IR photometry; 10 - color-excess; 11 - effective temperature in K, 12 - absolute visual magnitude, 13 - luminosity in solar units. The reddenings derived here are discussed in Sect. 3.2. We assume uncertainties of the $T_{\text {eff }}$ of $\sim 1000 \mathrm{~K}(2000 \mathrm{~K}$ in Gummersbach et al. 1995) and 0.2 dex in the luminosity (the same as in Gummersbach et al. 1995). The luminosity uncertainty for Hen S-59 is set to 0.3 dex (see Sect. 3). ${ }^{(a)} U B V$ data from Zaritsky et al. (2004) and $J H K$ data from 2MASS, ${ }^{(b)} U B V$ data from Ardeberg et al. (1972), ${ }^{(c)} U B V$ data from Massey (2002), ${ }^{(d)} U B V J H K$ data, reddening, and absolute visual magnitudes from Gummersbach et al. (1995).

The entire group of $\mathrm{B}[\mathrm{e}]$ supergiants in the Magellanic Clouds has not been studied closely, and its fraction of binaries is still uncertain.

Properties of $\mathrm{B}[\mathrm{e}]$ supergiants are similar to those of luminous blue variables (e.g., strong line emission and IR excess, close locations on the Hertzsprung-Russell diagram), and these groups are thought to be evolutionary connected (e.g., Clark et al. 2013). Another recent explanation of the $\mathrm{B}[\mathrm{e}]$ phenomenon in massive evolved stars involves mergers (Podsiadlowski et al. 2006). For example, this model explains the binary system R 4 as a merger from a triple system.

In such an uncertain situation it is important to search for more objects of this group. We attempted this by positionally cross-correlating the catalog of optical photometry of the LMC by Zaritsky et al. (2004) and the 2MASS catalog (Cutri et al. 2003). The selection criteria included slightly reddened colorindices of B-type stars and IR color-indices of $(J-H) \geq 0.3 \mathrm{mag}$ and $(H-K) \geq 0.7$ mag (Zickgraf et al. 1986). Objects found with these criteria were identified in the NOMAD $^{1}$ catalog (Zacharias et al. 2005). Initial results of this search were published in Miroshnichenko et al. (2011) and contained a list of ten objects whose optical and IR positions were separated by less than $3^{\prime \prime}$. It turned out that two of these objects positionally coincided with known B[e] supergiants Hen S-59 and Hen S-137 discovered by Gummersbach et al. (1995). Nevertheless, new observations of them are important, because results from only one observational epoch have been published so far.

Here we report the results of spectroscopic observations of four objects from the list by Miroshnichenko et al. (2011). The observations are described in Sect. 2. Analysis of the spectra and spectral energy distributions are presented in Sect. 3. Our findings are discussed in Sect. 4, and conclusions are given in Sect. 5.

\section{Observations}

The spectra were obtained with the Gemini South $8.1 \mathrm{~m}$ telescope located at Cerro Pachon (Chile) on 7 and 8 October 2011 as part of the Gemini Program ID GS-2011B-Q-81 with Hugo Levato as principal investigator. We used the Gemini MultiObject Spectrograph (GMOS) in the long-slit spectroscopic mode. The detector was an array of three $2048 \times 4608 \mathrm{EEV}$ chips with a pixel size of $13.5 \times 13.5 \mu \mathrm{m}$. The dark current

\footnotetext{
1 NOMAD is a merged version of USNO-B1.0 (Monet et al. 2003) and 2MASS (Cutri et al. 2003) catalogs.
}

was $\sim 3$ electrons per hour per pixel measured at $-113 \mathrm{C}$ at the Observatory. We use a grating with 1200 grooves per millimeter. We used CuAr comparison lamps for the wavelength calibration of the spectra. The spectral resolving power was $\sim 4400$ with a slit width of 0.5 . The spectral coverage was $\sim 4060-5570 \AA$ with two gaps around $\sim 4530 \AA$ and $\sim 5030 \AA$ due to connections between the CCD chips.

Four objects from the above list by Miroshnichenko et al. (2011) were observed. They are NOMAD 0196-0049799 (positionally coincides with ARDB 54, Ardeberg et al. 1972), NOMAD 0181-0125572 (hereafter N181), NOMAD 02030138943 (positionally coincides with Hen S-137), and NOMAD 0218-0100858 (positionally coincides with Hen S-59).

The spectra were reduced using IRAF ${ }^{2}$ with GMOS particular packages ${ }^{3}$. The standard procedure includes bias correction, cosmic ray cleaning, flatfielding, wavelength calibration and sky subtraction.

\section{Results}

Spectra of all the objects revealed the presence of Balmer and singly ionized permitted metallic lines (mostly Fe II). There are also emission lines of [FeII] (Figs. 1 and 2). Therefore, they all do exhibit the $\mathrm{B}[\mathrm{e}]$ phenomenon. Additionally, their $H-K$ colorindices are consistent with the CS dust presence (Fig. 4b), which accompanies the $\mathrm{B}[\mathrm{e}]$ phenomenon in most cases (Sect. 1).

Our analysis of the objects' spectral and photometric properties led to the following results as described below for each object separately. The photometric data along with our determinations of the total reddening (combined CS and interstellar in both the Milky Way and LMC, see discussion in Sect. 3.2) and absolute visual magnitudes based on the most recent distance toward LMC (50.0 $\pm 1.1 \mathrm{kpc}$, Pietrzyński et al. 2013) are presented in Table 1. Additionally, some other data for ARDB 54, Hen S-59, and Hen S-137 from literature are shown in Table 1 for comparison. The spectral energy distributions corrected for the derived reddening of the objects are shown in Fig. 3. Their

2 IRAF is distributed by the National Optical Astronomical Observatories, which is operated by the Association of Universities for Research in Astronomy, Inc., under a cooperative agreement with the National Science Foundation.

3 http://www .gemini .edu/sciops/data-and-results/ processing-software 
H. Levato et al.: New dusty B-type supergiants in the LMC
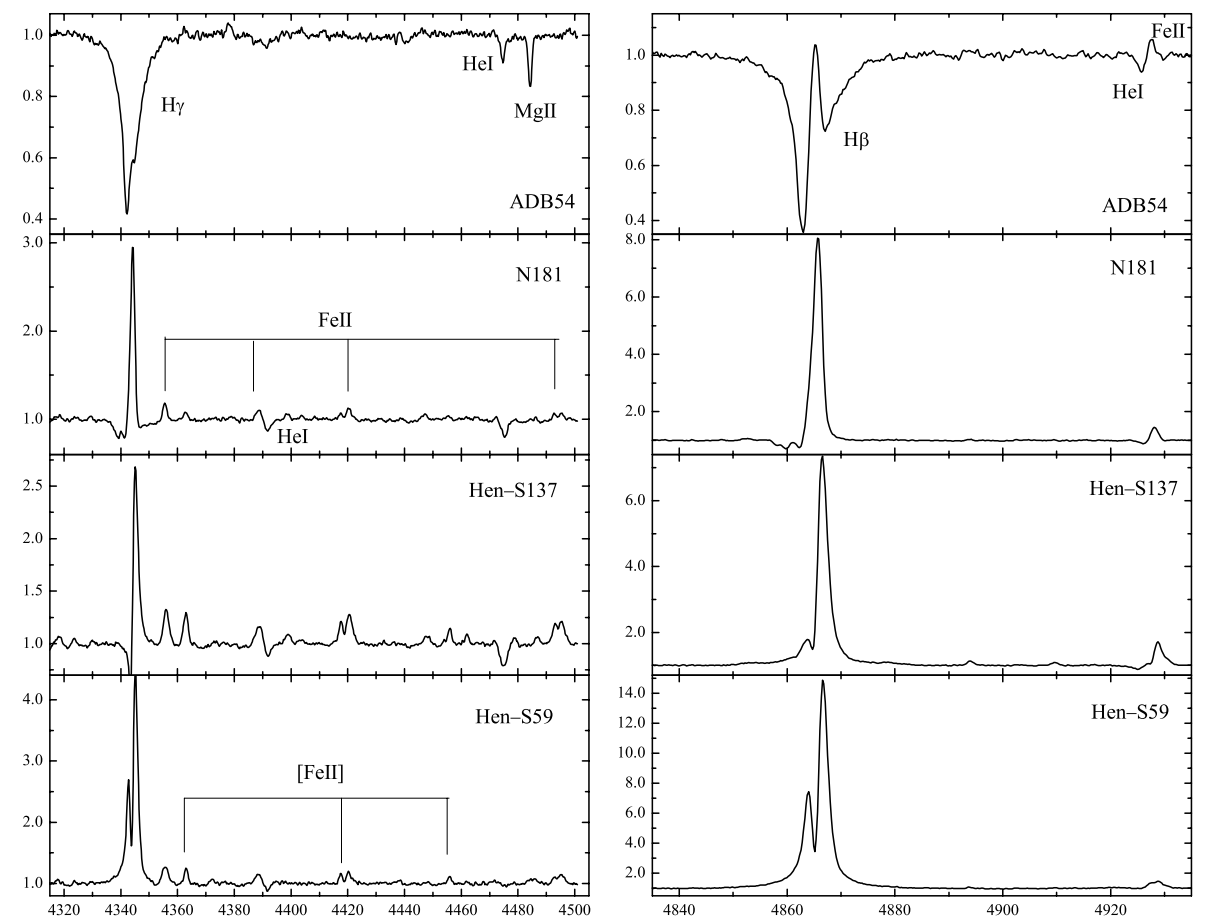

Fig. 1. Spectra of the four $\mathrm{B}[\mathrm{e}]$ supergiants in the $\mathrm{H} \gamma$ (left panel) and $\mathrm{H} \beta$ region (right panel). Wavelengths are geocentric; intensities are normalized to the underlying continuum.
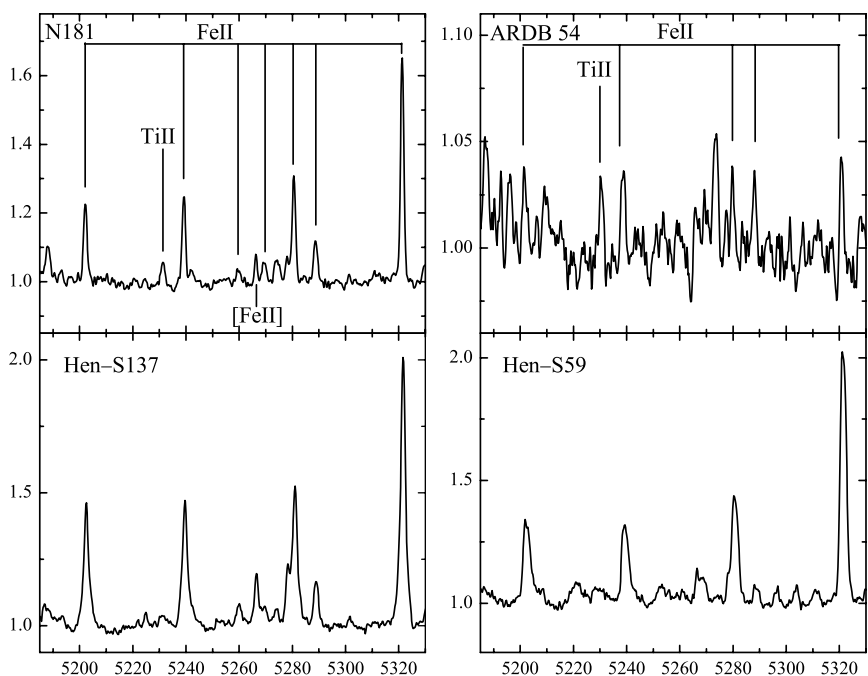

Fig. 2. The longest wavelength portions of our spectra of the four $\mathrm{B}[\mathrm{e}]$ supergiants. The lines shown are either Fe II or [Fe II], except those marked otherwise. Wavelengths and intensities are in the same units as in Fig 1.

optical and near-IR colors are shown in Fig. 4, and locations in the Hertzsprung-Russell diagram are presented in Fig. 5.

\subsection{Spectra}

$A R D B 54$. This object was first reported by Ardeberg et al. (1972), who suggested that it might be a multiple or an emissionline star based on its $U B V$ fluxes. Stock et al. (1976) took an objective prism spectrum of the star and classified it as B9 Ib. The optical color-indices (see Fig. 4a) as, a nearly equal strength of the He I $4471 \AA$ and the Mg II $4482 \AA$ lines (hereafter, we refer to the spectral line wavelengths in the Earth's rest frame) are consistent with this spectral type. This is the lowest temperature object in our sample, and it has the weakest emission-line
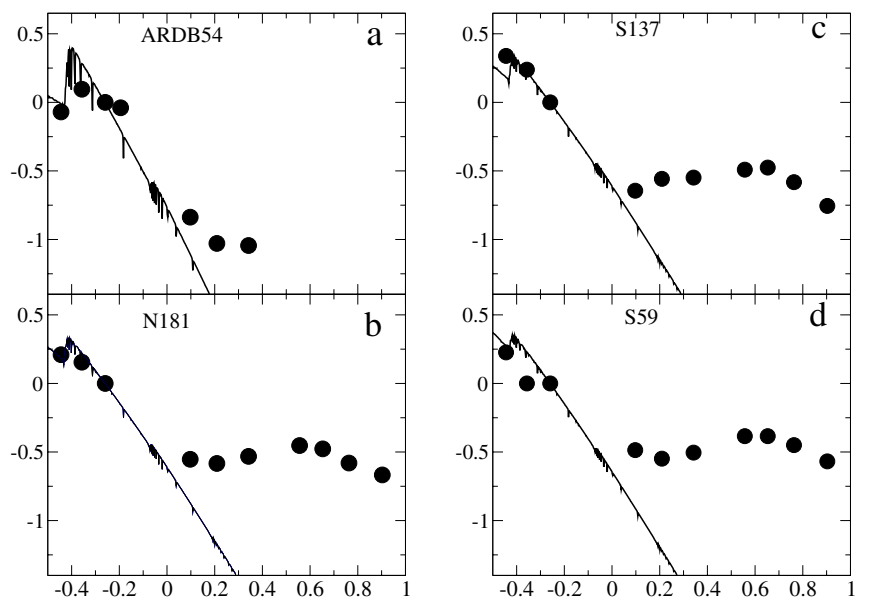

Fig. 3. Spectral energy distributions of the $\mathrm{B}[\mathrm{e}]$ supergiants. Panel a): ARDB 54, panel b): N181, panel c): Hen S-137, and panel d): Hen S-59. The optical UBV photometry for N181, Hen S-59, and Hen $\mathrm{S}-137$ is taken from Zaritsky et al. (2004); the optical UBVR photometry for ARDB 54 is taken from Massey (2002), the $J H K$ photometry is taken from the 2MASS catalog, and the 3.6-8.0 $\mu \mathrm{m}$ Spitzer photometry (the 4 longest-wavelength points) is taken from the SAGE survey data (Whitney et al. 2008). The fluxes are corrected for the reddening (see text). Solid lines represent theoretical SEDs from Kurucz (1993). We assumed $T_{\text {eft }}$ of $10000 \mathrm{~K}$ for ARDB 54, $16000 \mathrm{~K}$ for N181 and Hen S-137, and $19000 \mathrm{~K}$ for Hen S-59. The fluxes are normalized to the $V$-band flux and shown on a logarithmic scale versus logarithms of wavelengths in microns.

spectrum. Most of the emission lines are detected in the longestwavelength part of our spectrum between 5100 and $5500 \AA$, where we identified nearly 20 lines of Fe II, Ti II, Si II, and Cr II (Fig. 2). All of them do not exceed $10 \%$ of the continuum intensity at the peaks. The Fe II lines of multiplet 42 at $4923.92 \AA$ and $5018.43 \AA$ are blended with absorption lines of He I. Although our spectra were taken with a moderate resolution, the line radial velocities are consistent with each other. The average 

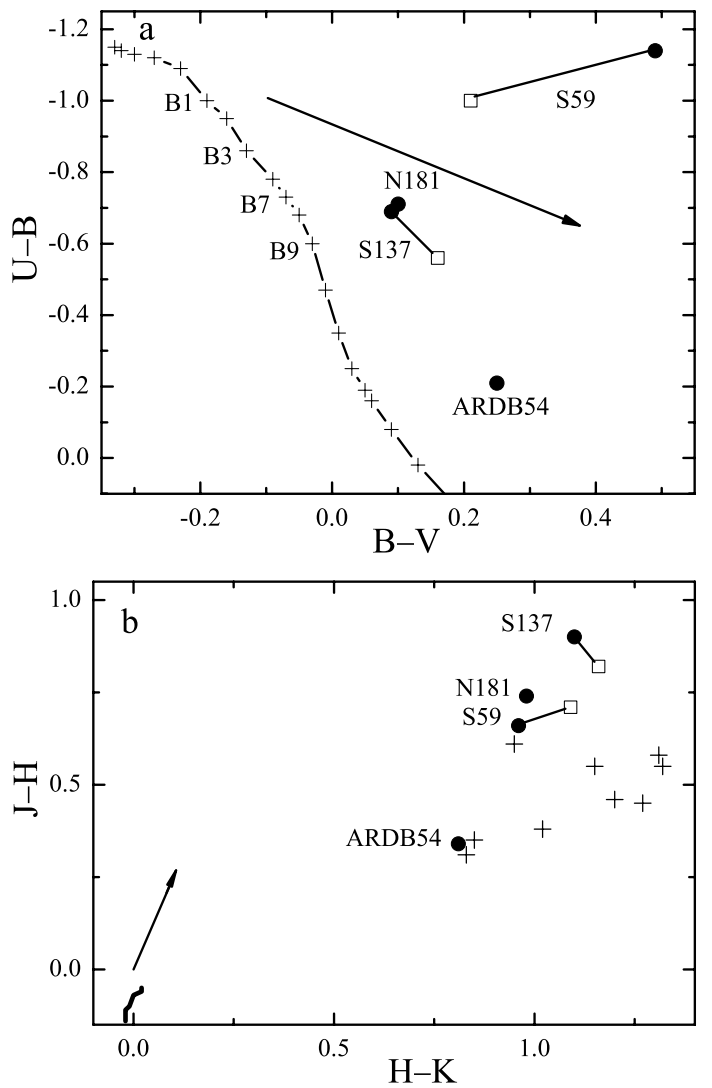

Fig. 4. Panel a): $\mathrm{A}(U-B) \sim(B-V)$ diagram. The solid line with pluses shows the intrinsic color-indices of Galactic supergiants (Straizhys 1977). Color-indices of the objects from Zaritsky et al. (2004) are shown by filled circles and those from Gummersbach et al. (1995) are shown by open squares. The line with an arrowhead represents an interstellar reddening vector for $E(U-B) / E(B-V)=0.65$, which is typical for both the Milky Way and the LMC. Panel b): A $(J-H) \sim(H-K)$ diagram for the known supergiants with the $\mathrm{B}[\mathrm{e}]$ phenomenon in the LMC (pluses) and the objects from Table 1 (the same symbols as in panel a). The solid line in the lower left corner represents intrinsic colors of B-type supergiants; the line with an arrowhead represents an interstellar reddening vector for $E(J-H) / E(H-K)=2.5$ (Wegner 1994).

heliocentric radial velocity for 14 unblended lines of Fe II, Ti II, and $\mathrm{Si}$ II in the spectrum of ARDB 54 is $216 \pm 7 \mathrm{~km} \mathrm{~s}^{-1}$.

Only one [Fe II] line at $5184.8 \AA$ was found. The latter does not mean that ARDB 54 exhibits no $\mathrm{B}[\mathrm{e}]$ phenomenon. It may be due to the following: a low effective temperature which suggests than the CS disk may not be fully ionized, a smaller amount of CS matter, which is also manifested by a smaller IR excess, as seen in Figs. 3 and 4, as compared to that around most other B[e] supergiants; and a limited wavelength range of our spectrum. The near-IR colors of ARDB 54 are indicative of the presence of CS dust (Fig. 4b). The latter gives additional support to the classification of ARDB 54 as a B[e] supergiant.

N181. This object has stronger emission lines than ARDB 54, although all the observed He I lines are seen in absorption. The Balmer line profiles exhibit a P Cyg type absorption but with an additional weak emission peak at $-275 \pm$ $5 \mathrm{~km} \mathrm{~s}^{-1}$ with respect to the main emission peak position. This secondary peak is most likely due to an interplay of a nearly edge-on orientation of the CS disk and a certain density distribution in it (Fig. 1). The blue edge of both $\mathrm{H} \gamma$ and $\mathrm{H} \beta$ is detected at $\sim-560 \mathrm{~km} \mathrm{~s}^{-1}$ from the main emission peak position, indicating a relatively high wind terminal velocity.

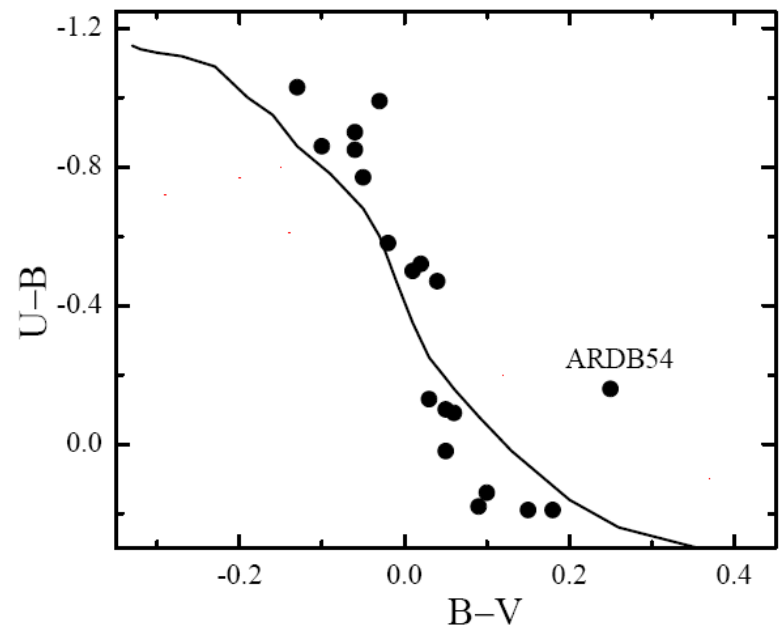

Fig. 5. A $(U-B) \sim(B-V)$ diagram for a region of the LMC within $20^{\prime}$ around ARDB 54. The photometric data are taken from Massey (2002). As in Fig. 4a, the solid line shows the intrinsic color-indices of Galactic supergiants.

We detected several [Fe II] emission lines in the spectrum of N181. They all are weaker than the detected Fe II lines. The strongest Fe II line at $5316 \AA$ reaches 1.6 of the continuum intensity at peak (Fig. 2). We identified one of the weak emission lines that seems to be unblended as that of $[\mathrm{N} \mathrm{II}]$ $5237.45 \AA$ (Fig. 2), but this identification requires confirmation with a higher-resolution spectrum. Overall, permitted metallic emission lines of N181 are somewhat narrower than those of the other observed objects. This allowed us to clearly detect two Mg I emission lines at 5172.68 and $5183.60 \AA$ (the third lines of this triplet at $5167.32 \AA$ seems to be blended with the Fe II $5169.03 \AA$ line). The average heliocentric radial velocity of $9 \mathrm{Fe}$ II lines in the spectrum of N181 is $255 \pm 4 \mathrm{~km} \mathrm{~s}^{-1}$. This value is within the 3- $\sigma$ uncertainty from those of other metallic lines (absorption He I and emission $\mathrm{Mg}$ I) and of the main emission peaks of Balmer lines.

Hen $\mathrm{S}-137$. The spectrum of Hen $\mathrm{S}-137$ is stronger and richer in emission lines than that of N181. Although $\mathrm{H} \gamma$ has a pure $\mathrm{P}$ Cyg profile, $\mathrm{H} \beta$ shows a double-peaked emission. This indicates a higher CS optical depth in Hen $\mathrm{S}-137$ and a similar viewing angle compared to N181. The blue edge of the $\mathrm{H} \gamma$ line is blueshifted by $\sim 380 \mathrm{~km} \mathrm{~s}^{-1}$ with respect to the emission peak. If this is not a projection effect, then the wind terminal velocity is lower than in N181, thus explaining a higher emission-line intensity due to a smaller CS density gradient. Nearly the same optical color-indices suggest similar temperatures and total reddenings for Hen S-137 and N181. The result that all the observed He I lines are in absorption is consistent with both effective temperature estimates shown in Table 1 for Hen $\mathrm{S}-137$.

The $5316 \AA$ Fe II line intensity at peak is nearly twice the underlying continuum intensity (Fig. 2). This is higher than that in the spectrum of $\mathrm{N} 181$ and comparable to that in the spectrum of Hen S-59. We identified 11 [Fe II] lines in the observed spectral region. At the same time, our spectrum shows weaker and slightly different Balmer line profiles compared to those presented in Gummersbach et al. (1995). The $\mathrm{H} \gamma$ line profile in Gummersbach et al. (1995) is double-peaked, while it has a P Cyg type in our spectrum. This difference is most likely due to a lower CS gas density at the time of our observations. At the same time, the average heliocentric radial velocity 
of 8 [Fe II] lines in our spectrum of Hen $\mathrm{S}-137$ is $258 \pm 7 \mathrm{~km} \mathrm{~s}^{-1}$, which is very close to $272 \pm 5 \mathrm{~km} \mathrm{~s}^{-1}$ reported for [Fe II] lines in the spectrum presented by Gummersbach et al. (1995).

Hen $S-59$. This object has the strongest Balmer lines in the sample, the strongest reddening, and the strongest UV excess. The $\mathrm{H} \beta$ line profile in our spectrum (see Fig. 1) has a similar peak intensity ratio compared to that published by Gummersbach et al. (1995), but our line intensity is stronger by a factor of $\sim 1.5$. Our $\mathrm{H} \gamma$ profile is also stronger and clearly double-peaked, while it has an irregular shape in the spectrum from Gummersbach et al. (1995). The latter may be due to a lower signal-to-noise ratio in their spectrum. The peaks of both $\mathrm{H} \beta$ and $\mathrm{H} \gamma$ are $170 \mathrm{~km} \mathrm{~s}^{-1}$ apart in our spectrum. The average heliocentric radial velocity of 8 [Fe II] lines in our spectrum of Hen S-59 is $249 \pm 10 \mathrm{~km} \mathrm{~s}^{-1}$, which is significantly different from $295 \pm 5 \mathrm{~km} \mathrm{~s}^{-1}$ reported for [Fe II] lines by Gummersbach et al. (1995).

Although the Balmer lines are stronger in our spectrum of Hen S-59 compared to those of Hen S-137 (see Fig. 1, the metallic lines are slightly stronger in our spectrum of the latter object. The IR excess is also a bit stronger in Hen S-137 (see Table 1), reflecting a larger amount of CS dust around it.

\subsection{Reddening and stellar parameters}

To correctly determine the objects' effective temperature and luminosity, both interstellar and CS reddening need to be taken into account. The rich emission-line spectra may affect the observed color-indices and introduce an additional optical and near-IR continuum. Since Gummersbach et al. (1995) have already addressed these problems for Hen S-59 and Hen S-137, we use some of their results and critically evaluate some others in the light of our new data.

Oestreicher et al. (1995) mapped the interstellar reddening in the foreground of the LMC and showed that it varies from $A_{V}=0$ to $0.15 \mathrm{mag}$ with an average of $0.06 \pm 0.02 \mathrm{mag}$. Using these results, Gummersbach et al. (1995) found that this part of the reddening toward Hen $\mathrm{S}-59$ and Hen $\mathrm{S}-137$ was $E(B-V)=$ 0.04 and $0.03 \mathrm{mag}$, respectively. The values are smaller than the color-excesses of any of our objects formally determined from their observed $(B-V)$ color-indices and intrinsic ones for any of the B-subtypes (see Fig. 4a). We only roughly estimate the foreground reddening and that within the LMC, because the interstellar extinction laws in the optical range are very close in both galaxies (e.g., Gordon et al. 2003).

Fundamental parameters of the $\mathrm{B}[\mathrm{e}]$ supergiants in the Magellanic Clouds were typically determined by fitting model spectral energy distributions to the IUE spectra and $U B V$ photometry (Zickgraf et al. 1986; Gummersbach et al. 1995). After the foreground extinction removal, the IUE data were used to determine the extinction within either the Large or the Small Cloud. This procedure assumes no CS contribution to the UV part of the spectrum and in all cases leads to a CS reddening of $\sim 0.2-0.3 \mathrm{mag}$ in the $B-V$ color-index. The latter seems natural due to the presence of the CS matter, but a CS contribution can also affect the UV part allowing for systematic errors in determining the fundamental parameters.

The amount of interstellar extinction within the LMC is not easy to derive. The interstellar dust distribution was described as patchy with $E(B-V)$ from 0 to 0.2 mag for stars in a range of the dereddened visual brightness of $V_{0}=12-14 \mathrm{mag}$ (Isserstedt 1975). Our inspection of the $U B V$ photometry of Btype stars around the studied objects (typically within $10^{\prime}-20^{\prime}$ ) from catalogs by Massey (2002) and Zaritsky et al. (2004) shows mostly small reddening of $E(B-V) \sim 0.1 \mathrm{mag}$. All the regions contain a few stars with $E(B-V)$ that are larger than those of our objects due to effects of both interstellar and CS matter. There are also stars with $U B V$ colors that are bluer than the intrinsic ones. This effect may be due to an unaccounted background emission (a high density of stars) or to the presence of unresolved stellar companions. A color-color diagram for stars around ARDB 54 is shown in Fig. 5 as an example. As seen in this plot, ARDB 54 is the most reddened star in this region that points to the presence of a CS reddening, which is still small to significantly alter the observed optical color-indices.

Based on the above analysis, we assume a total interstellar reddening for all the objects to be $E(B-V)=0.15 \mathrm{mag}$ (0.05 mag for the foreground component and $0.10 \mathrm{mag}$ for the one within the LMC). This approach sets the CS reddening at $E(B-V)=0.10 \mathrm{mag}$ for ARDB 54, N181, and Hen S-137. The CS reddening for Hen $\mathrm{S}-59$ is less certain because of its photometric variability discussed below.

A more consistent method of deriving both the reddening and fundamental parameters is to model the spectral line profiles and spectral energy distributions, but it has been shown that neither of the models explored so far (e.g., equatorial wind produced by bi-stability and Keplerian viscous disk) can explain the observed IR excesses satisfactorily (Porter 2003). Therefore, we rely on the dereddened optical color-indices and the spectral line content when estimating the stellar parameters.

Gummersbach et al. (1995) derived the fundamental parameters of Hen S-59 and Hen S-137 using the method described above. As one can see now, both objects show variations of their optical color-indices, which is not surprising for supergiants with strong winds. The case of Hen S-59 is very pronounced. It shows not only a large $U$-band excess in both optical data sets (see Table 1) but also a change in the optical color-indices that is inconsistent with the reddening vector (Fig. 4a). Since the IUE spectra discussed in Gummersbach et al. (1995) were taken about seven months after the optical photometry, fitting both sets of data to one model simultaneously may not be appropriate. The same suggestion may apply to Hen S-137, although the change of its color-indices is not that strong.

The simplest case of our four objects seems to be ARDB54. Its color-indices, although altered by a little CS reddening, are consistent with the ratio of the strengths of the He I $4471 \AA$ to the Mg II $4482 \AA$ line (see Sect. 3.1) and with very weak He I lines at 4120, 4143, and $4387 \AA$ (Lennon et al. 1993). The weak emission-line spectrum also implies a small contribution of the CS gas to the observed continuum.

The other three objects exhibit emission-line spectra of a similar excitation. In particular, the equivalent widths of the He I lines at 4120,4143 , and $4387 \AA$ are very similar. They also exhibit a weak Mg II $4481 \AA$ lines in emission. The He I $4471 \AA$ and the C II $4267 \AA$ lines look nearly the same in the spectra of N181 and He S-137 (equivalent widths of 0.55 and $0.13 \AA$, respectively). This is consistent with an early-B spectral type according to the data by Lennon et al. (1993) for Galactic B-type supergiants with a caution that our objects may have weaker absorption lines due to the CS contribution.

For N181, a combination of the spectrum and the optical color-indices suggests that its spectral type is B3-4 and an effective temperature of $T_{\text {eff }} \sim 16000-18000 \mathrm{~K}$. Close locations of N181 and Hen S-137 in the $(U-B) \sim(B-V)$ plane (Fig. 4a), along with similar spectral features suggest close 
effective temperatures. The observed optical color-indices imply a little contribution from the CS matter for these two objects.

Compared to N181 and Hen S-137, stronger Balmer lines in the spectrum of Hen S-59 may imply a higher effective temperature and/or a larger amount of CS gas. The former explanation seems to be more viable, because the He I $4471 \AA$ line in Hen $\mathrm{S}-59$ is the weakest of all in our sample that may be due to its partial filling with emission. The IR excess of Hen S-59 due to both its free-free/free-bound and dust emission is also weaker than those of N181 and Hen S-137 (see Fig. 3). Therefore, the spectrum of Hen S-59 is more consistent with an earlier B-subtype than it was suggested by Gummersbach et al. (1995). Helium lines typically turn into emission in stars earlier than B2 (e.g., Zickgraf et al. 1986; Miroshnichenko 2007). Therefore, we suggest that the spectral type of Hen $\mathrm{S}-59$ is B2-3 ( $\left.T_{\text {eff }} \sim 18000-20000 \mathrm{~K}\right)$.

\section{Discussion}

As shown above, the four observed objects have both emissionline spectra and IR excesses. They are located beyond the main-sequence on the Hertzsprung-Russell diagram (Fig. 5) and, therefore, are supergiants. Three of them (except for the brightest one, ARDB 54) were recently observed by the Spitzer Space Observatory in the course of the SAGE survey of the LMC in a spectral range of 3-8 $\mu \mathrm{m}$ (Whitney et al. 2008). These data are shown in Fig. 3, along with the previously obtained photometry. The 2MASS and SAGE data are consistent with each other and confirm the dusty nature of the IR excesses. Although the photometric data cover a relatively large range of wavelengths and the spectra were obtained with a high enough resolution, we attempt modeling of neither the spectral energy distributions nor the spectral line intensities because of a non-simultaneity of the data and the photometric and spectral variations discussed above.

The presence of the dusty IR excess allows some flexibility in interpretation. The object N181 (also known as SAGE 052747, van Aarle et al. 2011) was observed spectroscopically by the Spitzer Space Observatory (Gielen et al. 2011). The latter authors found a broad silicate emission feature at $10 \mu \mathrm{m}$ that is similar to those observed in post-AGB stars and classified N181 as such an object. Neither the optical photometry nor the optical spectrum were considered in this work. The conclusion was solely based on the IR data. However, an earlier Spitzer spectroscopy of two B[e] supergiants in the LMC, R66 and R126, by Kastner et al. (2006) showed silicate emission features very similar to those observed in the spectrum of N181 and post-AGB stars. Therefore, based on our optical spectrum and the derived fundamental parameters, we can suggest that N181 is much more likely a B[e] supergiant rather than a post-AGB object.

Our adjustments to the reddening and fundamental parameters of Hen S-59 and Hen S-137 changed their positions in the Hertzsprung-Russell diagram (Fig. 6). Nevertheless, both objects still reside in the post-main-sequence domain along with other supergiants that show the $\mathrm{B}[\mathrm{e}]$ phenomenon. With the new parameters, their initial mass is close to $25 M_{\odot}$ rather than to $\sim 10 M_{\odot}$ as suggested by Gummersbach et al. (1995). In this case the lower boundary for the luminosity of $\mathrm{B}[\mathrm{e}]$ supergiants with dusty IR excess in the Magellanic Clouds moves from $\log L / L_{\odot} \sim 4.0$ to $\sim 4.5$. The three dust-poor supergiants found by Graus et al. (2012) in the SMC have luminosities in the range $\log L / L_{\odot}=4.4-4.8$, which are near the lower boundary, but

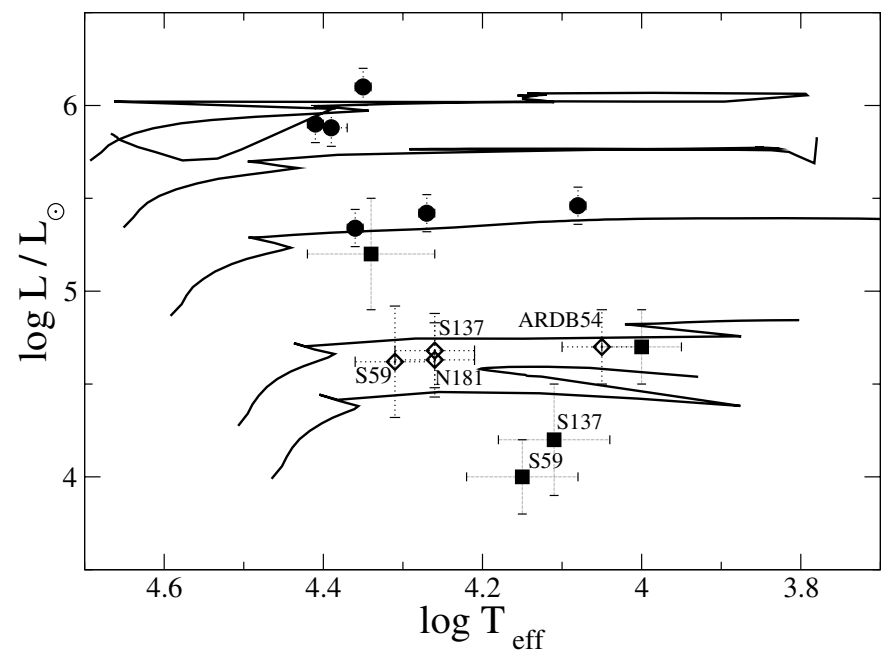

Fig. 6. A Hertzsprung-Russell diagram for supergiants with the $\mathrm{B}[\mathrm{e}]$ phenomenon in the LMC. The solid lines are the zero-age mainsequence and evolutionary tracks for single stars at the LMC metallicity from Schaerer et al. (1993). The tracks are shown for the initial masses of $15,25,40,60$, and $80 M_{\odot}$ from the lowest to the highest, respectively. Symbols: filled circles - objects from Zickgraf et al. (1986), filled squares - objects from Gummersbach et al. (1995) including Hen S-59 and Hen S-137 with fundamental parameters from that paper, and open diamonds - objects from this paper (see Table 1).

still consistent with it. Observations of fainter stars with IR excesses (e.g., from Miroshnichenko et al. 2011) should constrain this limit better.

A photometric monitoring of Hen S-59 as part of a gravitational lensing search program OGLE reported by Soszynski et al. (2009) resulted in finding periodic brightness variations in the I-band. The object's OGLE variable ID is LPV-83573, and its variations were detected with a period of 83.4 days and an amplitude of $\Delta I \sim 0.3 \mathrm{mag}$. This may be a manifestation of binarity of Hen $\mathrm{S}-59$. The object also exhibits variations of the $B-V$ color-index, 0.2 mag in Gummersbach et al. (1995) and 0.4 mag in Zaritsky et al. (2004), and a variable emission line radial velocity (see Sect. 3.1). Additionally, the strong UV excess ( $U-B \sim-1.0 \mathrm{mag}$ ) may indicate that the secondary companion is a fainter and hotter star. If such a companion does exist and contributes to the observed flux in the optical region, then both fundamental parameter sets derived by Gummersbach et al. (1995) in this paper will have to be reconsidered. Multicolor photometric monitoring is needed to clarify these issues.

\section{Conclusions}

We presented optical medium-resolution spectroscopy of four early-type stars from the LMC. Two of them, Hen S-59 and Hen $\mathrm{S}-137$, are recognized supergiants with the $\mathrm{B}[\mathrm{e}]$ phenomenon discovered by Gummersbach et al. (1995), who obtained their optical spectra nearly 20 years ago. Our new spectra confirm the presence of the $\mathrm{B}[\mathrm{e}]$ phenomenon in these objects and show a noticeable variability in the Balmer line profiles. Spectral parts redward of $\lambda=5200 \AA$ are presented here for the first time (see Fig. 2).

We re-estimated the fundamental parameters of Hen S-59 and Hen S-137 based on the line excitation in their optical spectra and compare with spectra of other similar objects. As a result, we assign a spectral type B2-3 to Hen S-59 and B3-4 to Hen S-137, instead of B5 and B6 in Gummersbach et al. (1995), 
respectively. This change along with the reddening adjustment moved these objects closer to the main-sequence and toward higher luminosities, implying a higher initial mass of $\sim 20 \mathrm{M}_{\odot}$ (see Fig. 6). Nevertheless, the new parameters have to be considered with caution, as the CS contribution to the observed colorindices and brightness is still uncertain.

Periodical variations of Hen S-59 discovered in the OGLE program (Soszynski et al. 2009) in combination with the UV excess and variable $B-V$ color-index and radial velocity may be signs of binarity of this object. So far only two binary B [e] supergiants are known in the Magellanic Clouds (Zickgraf 2006), but most of the Galactic objects of this type are recognized binaries (Wang et al. 2012).

The other two observed objects are ARDB 54 and N181. We confirm the spectral type of B9 I previously assigned to ARDB 54 (Stock et al. 1976) and show that it has an IR excess due to CS dust and the $\mathrm{B}[\mathrm{e}]$ phenomenon. We also found that the optical spectrum of N181 is very similar to those of Hen S-59 and Hen S-137. This similarity and the position on the Hertzsprung-Russell diagram prompts us to suggest that N181 is also a $\mathrm{B}[\mathrm{e}]$ supergiant and not a post-AGB object, as was concluded by Gielen et al. (2011).

Further spectral observations and contemporaneous photometric observations are needed to properly estimate the CS contribution to the objects' spectral energy distributions and refine their fundamental parameters. Observations of fainter objects (e.g., Miroshnichenko et al. 2011) are important to constrain the luminosity range of $\mathrm{B}[\mathrm{e}]$ supergiants in the Magellanic Clouds and to search for less luminous counterparts (FS CMa type objects) that have only been recognized in the Milky Way so far.

\section{References}

Allen, D. A., \& Swings, J.-P. 1976, A\&A, 47, 293

Ardeberg, A., Brunet, J. P., Maurice, E., \& Prevot, L. 1972, A\&AS, 6, 249

Clark, J. S., Bartlett, E. S., Coe, M. J., et al. 2013, A\&A, 560, A10

Conti, P. S. 1976, General Discussion in Be and Shell Stars, ed. A. Slettebak (Dordrecht, Holland: IAU), IAU Symp. 70, 447

Cutri, R. M., Skrutskie, M.F., Van Dyk, S., et al. 2003, CDS/ADC Collection of Electronic Catalogues, Vizier On-line Data Catalog: II/246
Gielen, C., Bouwman, J., van Winckel, H., et al. 2011, A\&A, 533, A99 Gordon, K. G., Clayton, G. C., Misselt, K. A., Landolt, A. U., \& Wolff, M. J. 2003, ApJ, 594, 279

Graus, A. S., Lamb, J. B., \& Oey, M. S. 2012, ApJ, 759, 10

Gummersbach, C. A., Zickgraf, F.-J., \& Wolf, B. 1995, A\&A, 302, 409

Isserstedt, J. 1975, A\&A, 41, 175

Kastner, J. H., Buchanan, C. L., Sargent, B., \& Forrest, W. J. 2006, ApJ, 638, L29

Kurucz, R. L. 1993, Smithsonian Astr. Obs. CD-ROM No. 19

Lamers, H. J. G. L. M., Zickgraf, F.-J., de Winter, D., Houziaux, L., \& Zorec, J. 1998, A\&A, 340, 117

Lennon, D. J., Dufton, P. L., \& Fitzsimmons, A. 1993, A\&AS, 97, 559

Massey, P. 2002, ApJS, 141, 81

Miroshnichenko, A. S. 2007, ApJ, 667, 497

Miroshnichenko, A. S., Manset, N., Kusakin, A. V., et al. 2007, ApJ, 671, 828

Miroshnichenko, A. S., Manset, N., Polcaro, F., et al. 2011, in Active OB stars: structure, evolution, mass loss, and critical limits, eds. C. Neiner, G. Wade, G. Meynet, \& G. Peters, Proc. IAU Symp., 272, 260

Monet, D. G., Levine, S. E., Canzian, B., et al. 2003, AJ, 125, 984

Oestreicher, M. O., Gochermann, J., \& Schmidt-Kaler, T. 1995, A\&AS, 112, 495

Pietrzyński, G., Udalski, A., Poleski, R., et al. 2013, Nature, 495, 76

Podsiadlowski, P., Morris, T. S., Ivanova, N. 2006, in Stars with the B[e] Phenomenon, eds. M. Kraus, A. S. Miroshnichenko (San Francisco: ASP), ASP Conf. Ser., 355, 259

Porter, J. M. 2003, A\&A, 398, 631

Schaerer, D., Maeder, A., Meynet, G., \& Schaller, G. 1993, A\&AS, 98, 523

Stahl, O., Smolinski, J., Wolf, B., \& Zickgraf, F.-J. 1989, in Physics of Luminous Blue Variables, eds., K. Davidson, A. F. J. Moffat, \& H. J. G. L. M. Lamers, (Dordrecht: Kluwer), 295

Soszynski, I., Udalski, A., Szymanski, M. K., et al. 2009, Acta Astron., 59, 239 Stock, J, Osborn, W., \& Ibanez, M. 1976, A\&AS, 24, 35

Straizhys, V. 1977, Multicolor Photometry of Stars (Vilnius: Mokslas Publ.)

van Aarle, E., van Winckel, H., Lloyd Evans, T., et al. 2011, A\&A, 530, A90

Wang, Y., Weigelt, G., Kreplin, A., et al. 2012, A\&A, 545, L10

Wegner, W. 1994, MNRAS, 270, 229

Whitney, B. A., Sewilo, M., Indebetouw, R., et al. 2008, AJ, 136, 18

Zacharias, N., Monet, D. G., Levine, S. E., et al. 2005, Vizier On-line Data Catalog: I/297

Zaritsky, D., Harris, J., Thompson, I. B., \& Grebel, E. 2004, AJ, 128, 1606

Zickgraf, F.-J. 2006, in Stars with the B[e] Phenomenon (San Francisco: ASP), eds. M. Kraus, A. S. Miroshnichenko, ASP Conf. Ser., 355, 135

Zickgraf, F.-J., Wolf, B., Stahl, O., Leitherer, C., \& Klare, G. 1985, A\&A, 143, 421

Zickgraf, F.-J., Wolf, B., Leitherer, C., Appenzeller, I., \& Stahl, O. 1986, A\&A, 163,119

Zickgraf, F.-J., Wolf, B., Stahl, O., \& Humphreys, R. M. 1989, A\&A, 220, 206

Zickgraf, F.-J., Stahl, O., \& Wolf, B. 1992, A\&A, 260, 205 